\title{
Aprendizagem Centrada no Participante ou no Professor? Um Estudo Comparativo em Administração de Materiais
}

\section{Participant or Professor Centered Learning? A Comparative Study in Applied Social Sciences}

\author{
Waldemar Hazoff Júnior* \\ Mestre pela FEA/USP \\ Professor de Administração da UniFMU, São Paulo/SP, Brasil.
}

Antonio Carlos Aidar Sauaia

Doutor em Administração pela FEA/USP.

Professor Associado da FEA/USP, São Paulo/SP, Brasil.

*Endereço: Rua Paulo Franco, 679, São Paulo/SP, 05305-031.E-mail: whazoffjr@terra.com.br 


\section{ResUMo}

A carga quantitativa presente em disciplinas de Administração tem provocado desconforto nos alunos que buscavam conteúdos humanistas, ao optarem pelas Ciências Sociais Aplicadas. O desconforto se agrava ainda mais quando a disciplina é oferecida às sextasfeiras no período noturno, comprometendo-se a aprendizagem. Formatou-se um estudo com quatro turmas de alunos (matutino e noturno) em duas instituições privadas, onde a disciplina Administração de Materiais e Bens Patrimoniais foi ministrada segundo dois procedimentos: $\mathrm{P}_{1}$ centrado no professor e $\mathrm{P}_{2}$ centrado nos alunos. Ambos fizeram uso das mesmas atividades encadeadas de maneira distinta, alternando-se aulas expositivas centradas no professor, exercícios centrados nos alunos e testes objetivos para avaliação de desempenho. $\mathrm{O}$ tratamento estatístico dos dados comprovou efeitos significativamente distintos nos procedimentos adotados, com desempenho superior (significância de 1\%) na abordagem centrada nos alunos em ambas as instituições (IES). Conclui-se que além da atitude positiva e determinada do docente, a simples escolha de um procedimento menos centrado no professor e mais orientado para os alunos pode aumentar a eficiência do processo educacional, sem que sejam necessários gastos adicionais.

Palavras-chave: aprendizagem centrada no participante; administração de empresas; ciências sociais aplicadas.

\section{Abstract}

The quantitative approach in the Business courses has distressed students searching for humanistic contents in Applied Social Sciences area of knowledge. One can observe this in the Materials and Patrimonial Properties Management course taught during the Business Administration program. This situation becomes still worse when the course is offered on Fridays evenings. This study dealt with four classes (morning and evening sessions) in two private institutions. The course was taught according to two procedures: a) professor centered, and b) participant centered. The same activities were scheduled in a distinctive way. The results indicated that the students' achievement were (statistically significant at $1 \%$ ) superior in the participant centered procedure $\left(\mathrm{P}_{2}\right)$ in comparison to the professor centered procedure $\left(\mathrm{P}_{1}\right)$. Very low investments for designing and planning the didactic materials made possible to produce better achievement of the students.

Key words: participant centered learning; business administration; applied social sciences. 


\section{INTRODUÇÃO}

A sociedade complexa torna cada vez mais evidente a necessidade de formação e capacitação para o mercado de trabalho. A diversidade de opções, a fragilidade do conhecimento e a responsabilidade social têm exigido maior empenho dos atores.

No Brasil, setores da sociedade têm demonstrado preocupação com os aspectos sociais e com o desenvolvimento de políticas de inclusão social. As instituições de ensino, em todos os níveis, procuram assimilar as mudanças, desenvolvendo modelos e práticas que permitam contribuir com este processo. As instituições privadas, em particular, concentram esforços em captar alunos para sustentar sua atividade. Estes buscam sua capacitação profissional e o ingresso no mercado de trabalho, mais pelo diploma que pelo conteúdo assimilado.

Este modelo tem promovido o acesso a IES de alunos com falhas de formação e que não estão comprometidos ou motivados com a carreira pela qual optaram. Pesquisas junto a egressos de Instituições de Ensino Superior [IES] revelam que muitos alunos não se identificam com o curso que fizeram ou entendem que este é deficiente, ou então o concluíram para a simples obtenção do título (Carneiro, 2002).

Pesquisas revelam que parcela significativa dos professores de terceiro grau chega ao magistério sem preocupação mínima com aspectos didáticos e pedagógicos (Chaves, 2003). Para Chagas (2002, pp. 14-18) o ensino do terceiro grau apresenta peculiaridades que o associam a uma linha específica de pesquisa. O professor universitário vive uma realidade diferente em relação a seus pares do ensino médio e fundamental. Eckstein (1982) destacava que, ao contrário dos professores de outros níveis de ensino, o nível superior não recebia nenhuma formação didática específica. Poder-se-ia lecionar sem o conhecimento prévio dos pressupostos psicológicos, didáticos e sociais relacionados ao aprendizado, induzindo uma visão míope. Paradoxalmente, o professor universitário leciona para adultos, muitos deles com experiência profissional, que já não aceitam passivamente certas colocações. Segundo Cavalcanti (1999), estudantes universitários não são exatamente adultos, mas estão próximos desta fase de suas vidas. $\mathrm{O}$ ensino centrado no professor pode resultar, para muitos deles, num retardamento da maturidade, pois coloca alunos em total dependência dos professores e currículos estabelecidos, não se estimulando nem apoiando eventuais iniciativas. A instituição e o professor decidem o que, quando e como os alunos 
devem aprender cada assunto ou habilidade, adaptando-se às regras preestabelecidas.

Apesar dos avanços recentes na qualificação do corpo docente, os professores em geral continuam desatentos aos aspectos curriculares e pedagógicos (Steffen, 1997) e repetem os padrões intuitivos e as abordagens tradicionais (Cardoso, 1998). Em faculdades de engenharia o método educacional é centrado no professor, sendo principal transmissor de conhecimentos. Ele determina o nível, o conteúdo, o ritmo, o método e o tipo de avaliação. A relação entre professor e aluno é vertical (Kuri, 1993), cabendo ao professor a maior parte das iniciativas.

Entendendo que os encontros ANPAD representam um ambiente para apresentação, avaliação e debate preliminar de material para publicação nas principais revistas brasileiras de administração, constata-se que em 2005, dos 37 trabalhos apresentados na categoria ensino e pesquisa em administração, 25 trataram da questão do ensino. Deste subgrupo, 9 trataram objetivamente de métodos, propondo métodos alternativos, fazendo cotejos comparativos entre métodos, desenvolvendo instrumentos educacionais, tais como simuladores que permitam centrar o processo educacional no participante e analisar métodos mais adequados a perfis de estudantes. Neste universo de autores, apenas um estava vinculado a uma instituição privada, sendo os demais de instituições federais ou estaduais. Em 2006, dos 38 trabalhos apresentados, 7 orientaram-se ao estudo de métodos educacionais. Nota-se a preocupação dos autores com a necessidade de inovar o processo educacional e que esta inovação pode ser propiciada pelo uso combinado de métodos educacionais. Todos os autores estão vinculados a universidades públicas. Esta quantidade expressiva sugere que o cenário descrito por Kuri (1993) vem mudando, percebendo-se movimentos de pesquisa para desenvolver, aprimorar e adequar métodos que, devidamente utilizados, podem contribuir para a melhoria do processo educacional. Martins e Passos (2003) destacam que ainda são escassas as pesquisas teórico-empíricas sobre métodos educacionais nas áreas de administração e contabilidade.

Algumas disciplinas do curso de Administração têm elevada carga quantitativa, o que provoca desconforto em alunos que optam pelas Ciências Sociais Aplicadas. Este é o caso da disciplina de Administração de Materiais e Bens Patrimoniais, que se agrava ainda mais quando oferecida às sextas-feiras no período noturno. A prática docente revela que alunos de ciências sociais aplicadas preferem modelos e ferramentas qualitativas a quantitativas. Freqüentemente expressam sua dificuldade em utilizar e interpretar modelos matemáticos, causando o referenciado desconforto. É proposta deste artigo discutir as possibilidades de aprendizagem 
que surgem para contornar esta dificuldade manifesta pelos estudantes. Não se pretendeu, de modo algum, evitar os conteúdos quantitativos. Procurou-se trabalhar os modelos matemáticos em situações-problema, em que os resultados numéricos tivessem maior significado. A sexta-feira foi destacada pela dificuldade de aprendizagem em função do cansaço do aluno, o que afeta o nível de concentração e a capacidade de raciocínio. Pretende-se verificar se, nesta condição adversa, um método alternativo pode ser contributivo.

Formatou-se este estudo com quatro turmas de alunos (matutino e noturno) em duas instituições privadas (IES), onde o conteúdo oficial da disciplina foi ministrado segundo dois procedimentos distintos $\left(\mathrm{P}_{1}\right.$ e $\left.\mathrm{P}_{2}\right)$, usando-se das mesmas atividades encadeadas segundo lógicas diferentes (Kolb, 1984). Em todas as turmas cumpriuse o mesmo programa, utilizaram-se os mesmos instrumentos de avaliação, sem prejuízo de conteúdos programáticos. Foram testados dois encadeamentos de atividades, verificando-se os resultados produzidos. Os procedimentos de cálculos para a resolução dos problemas propostos foram apresentados na íntegra nos dois procedimentos (vide Tabela 2).

\section{Gestão de Materiais}

Estudaram-se segundo um critério temporal, as contribuições de diferentes autores da área. Observou-se que a área de materiais ampliou seu horizonte operacional para uma dimensão estratégica (Gaither \& Frazier, 2002; Slack, Chambers, Harland, Harrison, \& Johnston, 1999).

Livros didáticos desta área de conhecimento apresentaram como propostas de planejamento de recursos materiais: a) o uso de lotes econômicos de compras; b) a elaboração de estrutura de produto; e c) a definição de lista de compras (Burbidge, 1981; Eilon, 1962; Magee, 1958; Ramlow \& Wall, 1967; Scheele, Westerman, \& Wimmert, 1964).

Em 1982 Bedworth e Bailey (pp. 108-111) discutem um sistema de planejamento específico para recursos materiais, denominado Manufacturing Requirements Planning [MRP]. Vollmann, Berry e Whybark (1984), Plossl (1985), Bertrand, Wortmann e Wijngaard (1990), entre outros, apresentaram o MRP como parte de um sistema de Planejamento de Recursos de Manufatura (Manufacturing Requirements Planning [MRP] II). Os estudos avançaram, destacando-se suas contribuições à gestão de materiais (Gaither \& Frazier, 2002; Higgins, Roy, \& Tierney, 1996; Morton \& Pentico, 1993; Vollmann, Berry, \& Whybark, 1997; Wohl, 1995). 


\section{Componentes do MRP}

O sistema MRP está dividido em seis arquivos básicos, como se explicita em seguida.

\section{Lista de materiais}

Para Dias (1997, p. 133) e Moreira (1996, p. 531) lista de materiais é um arquivo destinado a especificar e determinar as quantidades exatas de matériasprimas, componentes e sub-rotinas adotadas na elaboração e confecção do produto final, identificando relações de dependência entre materiais e produto final representadas na estrutura de produto.

\section{Plano Mestre de Produção (Master Program Schedule [MPS]).}

O MPS é montado para recompor estoques ou atender a pedidos de clientes. A viabilidade de cada proposta de produção deve ser testada nos subsistemas que a apóiam. Materialmente, determinada proposta de produção, declarada no MPS, só ocorrerá se na data programada estiverem disponíveis recursos materiais nas quantidades necessárias. Sendo inviável, programa-se outra data (Gaither \& Frazier, 2002, pp. 312-313).

\section{Demanda de produto}

A montagem do MPS necessita das previsões de demanda e pedidos em carteira de produto acabado. Para Slack et al. (1999, p. 445), Gaither e Frazier (2002, p. 313) e Dias (1997, p. 132), a área de vendas gerencia uma carteira de pedidos dinâmica e flutuante, composta por pedidos confirmados de clientes, os chamados pedidos em carteira e uma parcela especulativa gerada a partir da avaliação de dados históricos.

\section{Registros de inventários}

Correa, Gianesi e Caon (1999, p. 101) defendem que, no momento da programação, é imperativo ter informações verdadeiras e disponíveis (exatidão de estoque). Registros errados de quantidades, ou não disponíveis, introduzem desconfiança para usuários do sistema quanto à sua capacidade de prover a necessidade material de modo adequado.

\section{Previsão de demanda}

A discussão das melhores técnicas de previsão não é conclusiva. Para Moreira (1996, pp. 318-349), Gaither e Frazier (2002, pp. 54-87) e Slack et al. (1999, pp. 
711-712), métodos de previsão de demanda dependem da disponibilidade de dados, tempo e recursos. Correa et al. (1999, pp. 236-253), Martins e Campos (2000, p. 96) associam a escolha adequada do instrumento de previsão ao horizonte pretendido. Projeções de curto prazo podem ser amparadas por técnicas matemáticas como média móvel, média móvel ponderada e suavizamento exponencial. Previsões de longo prazo exigem métodos que contemplem as mudanças do ambiente neste período.

Moreira (1996, p. 349) recomenda, admitindo exceções, o uso de métodos qualitativos e causais para previsões de médio e longo prazo e análise de séries temporais para curto prazo.

Dias (1997, pp. 28-40), Moreira (1996, pp. 317-349), Gaither e Frazier (2002, pp. 55-80) e Slack et al. (1999, pp. 707-712) identificam incerteza nos métodos vigentes, sugerindo composição de técnicas. Propõem o uso de técnicas quantitativas não causais como regressão linear simples aplicada à série histórica e técnicas causais como regressão linear múltipla para estabelecer correlações que considerem ambiente econômico, sazonalidade e ações da concorrência.

6. Aplicação de lote econômico de compras no processo de tomada de decisão

Gaither e Frazier (2002, pp. 270-273) e Slack et al. (1999, pp. 380-407) defendem o uso de Lotes Econômicos de Compra [LEC] para orientar decisões de quanto comprar e quando repor estoques, recomendando esta prática para empresas com demanda estável no tempo.

Clientes e fornecedores, ao compartilharem informações em tempo real, viabilizam redução de estoques no sistema (Gaither \& Frazier, 2002, p. 301), sendo recomendável o uso combinado de esforços logísticos e modelos matemáticos de decisão para adicionar valor nas operações de compras (Correa et al., 1999, pp. 60-69).

\section{O Processo Educacional}

Para Brown e Atkins (1997) os professores universitários são funcionalmente responsáveis pelo ensino, pesquisa e gerenciamento do processo educacional. Cabe-lhes gerenciar a escolha de métodos adequados que permitam captar a atenção do público alvo por um período adequado de tempo, de modo oportuno e encaminhando-os a tarefas apropriadas.

Gage (como citado em Moreira, 1997, p. 25) conceitua método de ensino e 
estratégia de ensino como sinônimos. Etimologicamente, método significa caminho até a consecução de algo, um meio para alcançar objetivos determinados.

Wang, Haertel e Walberg (1990, pp. 30-43), Fouts e Myers (1992, pp. 356361), Bujold e Saint-Pierre (1996, pp. 75-79) e Keller e Hirsch (1998, pp. 1-9) afirmam que, independente do método, o professor, ao gerenciar as atividades propostas em sala de aula, deve utilizar instrumentos didáticos que intensifiquem a participação do aluno no processo de aprendizagem, compartilhando interesses e experiências. Ao definir uma estratégia de ensino, o professor deve alinhar a preferência dos alunos e suas habilidades pessoais para a condução do processo (Libâneo, 2004, p. 6).

Brown e Atkins (1997) propõem o modelo representado na Figura 1, estabelecendo um gradiente entre procedimentos de ensino e nível de participação e controle dos atores no processo educacional.

\section{Figura 1: Gradiente de Participação e Controle}

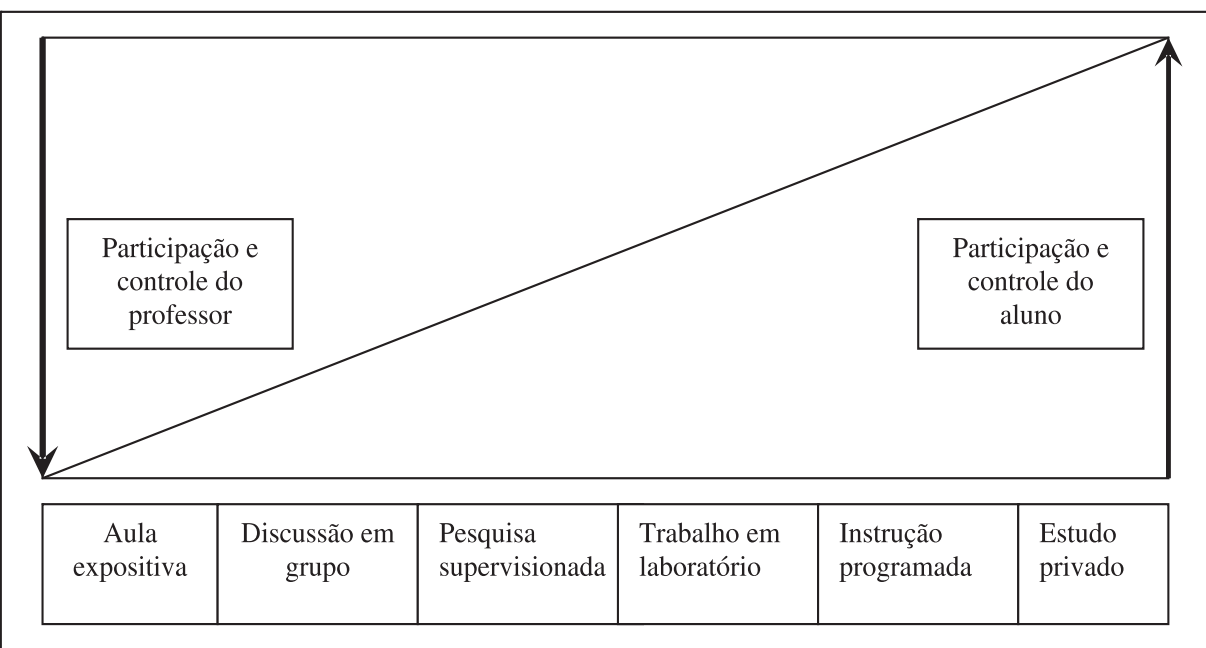

Fonte: Brown e Atkins (1997, p. 76).

Eckstein (1982), Gibbs (1995), Rosenshine (1995), Libâneo (2004) e Pretto (2006) propõem o uso de métodos centrados no aluno como forma de enriquecimento das aulas e formação de pessoas capazes de participar da vida social, econômica e política de uma sociedade moderna, tornando-se sujeito ativo na elaboração do conhecimento, amparado no professor que assume a função de facilitador neste processo de apropriação do conhecimento. 
Becker (1993, p. 9) e Pretto (2006, pp. 31-34) ponderam que a pedagogia centrada no professor tende a valorizar relações hierárquicas que, em nome da transmissão do conhecimento, podem produzir ditadores. Ao colocar o aluno no centro do processo, comete-se o erro de atribuir a este o domínio do conhecimento sistematizado em determinada área, capacidade de abstração, capacidade de concentração e estruturação do conhecimento (Saroyan \& Snell, 1997).

Anastasiou (1998, p. 39) e Chagas (2002, pp. 64-70) afirmam ser a aula expositiva o principal e mais antigo instrumento de ensino no Brasil em todos os níveis; pesquisas para complementá-la ou substituí-la não lograram sucesso pleno.

Feinstein (2001, pp. 432-433), Sauaia (1995) e Teixeira (1995) entendem que, apesar de eficaz, a aula expositiva mostra-se pouco eficiente, pois ativa apenas o sentido da audição. Eventos simulados e atividades vivenciais, devidamente estruturadas, permitem assimilar princípios que são integrados em novas situações e nelas aplicados.

Pfeiffer e Jones (1980, pp. 21-22) sugerem que aprendizagem vivencial ocorre quando uma pessoa se envolve em dada atividade, analisa-a criticamente, extraindo algum insight útil desta análise e gerando capacidade de aplicação dos resultados. Para Moran e Behrens (2000, pp. 27-29) vivência é uma cópia simplificada e dinâmica da realidade em que se pretende desenvolver a aprendizagem.

Segundo Feinstein (2001, pp. 423-426), propostas vivenciais possibilitam aprendizagem, sendo que a simulação tem demonstrado aspectos positivos, quando comparados com outras atividades vivenciais (Driscoll, 1994; Towne, Jong, \& Spada, 1993). Tansey (como citado em Sauaia, 1995, p. 67) considerava que simular é essencialmente fazer de conta. Esta condição, quando apoiada por um roteiro preestabelecido, permite colocar os participantes como agentes, responsáveis pela definição de rumos para a situação simulada proposta.

\section{A VAliaÇÃo}

Para Luckesi (1990) a ação de avaliar é relacionada a determinar, verificar, reconhecer, fazer idéia, calcular, apreciar, ajuizar ou refletir sobre algo, sendo formulada a partir de métodos de atribuição de valor ou qualidade a alguma coisa, ato, ou ação em curso; avaliação demanda posicionamento a favor ou contra o que está sendo avaliado.

Méndez (2002) explica que a técnica de avaliação é o meio utilizado para obter informações, e o instrumento de avaliação o recurso utilizado. Ao selecionar 
técnicas e instrumentos de avaliação devem-se considerar, entre outros, os objetivos pretendidos, as condições de trabalho, o tempo do professor e o número de alunos.

Tyler (1989, p. 35) entende que avaliação é a última atividade do processo educacional, possibilitando verificar a intensidade com que os objetivos educacionais foram atingidos por programas curriculares e instrucionais. Este tipo de proposta de avaliação é conhecido como avaliação somativa.

Zindeluk (como citado em Chaves, 2003, p. 19) identifica Cronbrach no artigo Courus improvement through evaluation publicado em 1963, como a primeira a afirmar que avaliação é útil quando realizada durante o processo educacional, permitindo em tempo real a sua correção. Scriven (como citado em Saul, 1988, p. 26) acreditava que estabelecer objetivos prévios, conforme propunha Tyler, restringia o programa somente ao previsto, impedindo a configuração de novos objetivos. Para trazer flexibilidade, propunha a montagem de um protocolo de intenções, a ser adaptado ao longo do processo.

O uso de avaliações formativas, efetuadas no decorrer do processo, com o intuito de aperfeiçoá-lo, comunica a professores e alunos a sua evolução e o nível com que os objetivos preestabelecidos estão sendo atingidos. Por meio do acompanhamento procura-se identificar ações que promovam o aprendizado.

Bloom (1956) defende que qualquer proposta de ensino e avaliação deve estabelecer, de forma clara e precisa, seus objetivos. Para tanto preconiza, além das avaliações formativa e somativa, propostas por Scriven, o uso de avaliação diagnóstica. Esta identifica no início do processo educacional como o aluno se encontra em relação ao assunto a ser trabalhado e permite descobrir variáveis que possam afetar o processo. Com esses três tipos, Bloom (1956) amplia o conceito de avaliação, inserindo-o no conjunto da prática educativa, passando a influenciar o processo de ensino.

\section{Objetivos da Pesquisa}

Três elementos influenciaram a elaboração deste estudo:

1) A carga quantitativa presente na disciplina de Administração de Materiais e Bens Patrimoniais provoca desconforto aos participantes.

2) Parte dos estudantes de administração orienta a carreira para empresas de serviços não considerando relevantes as contribuições desta disciplina para sua formação. 
3) A necessidade de melhorar a assimilação de conteúdos dos alunos que freqüentam a disciplina.

O desconforto declarado pelos alunos de Administração para calcular e interpretar resultados motivou este estudo, que compara dois métodos e verifica as diferentes contribuições. Todos os tópicos previstos no conteúdo programático foram contemplados nos dois procedimentos. Foram usados os mesmos instrumentos de avaliação para ambos os procedimentos $\mathrm{P}_{1}$ e $\mathrm{P}_{2}$, assegurando-se a integridade da abordagem quantitativa prevista no programa original.

Para cumprir tal propósito, selecionou-se atividade empresarial que evidencia a produção de bens tangíveis e intangíveis simultaneamente (restaurante). Para esta atividade elaboraram-se situações estruturadas que contemplam o conteúdo programático da disciplina (gestão de um restaurante simulado), comparando a aprendizagem proporcionada por um procedimento centrado no professor $\left(\mathrm{P}_{1}\right) \mathrm{e}$ um procedimento centrado no aluno $\left(\mathrm{P}_{2}\right)$. Vale destacar que a pesquisa realizada por Morilha, Muritiba e Casado (2005), em faculdade privada de Administração da cidade de São Paulo, revelou que os alunos preferiam métodos educacionais ativos, aqueles que colocam os estudantes no centro do processo educacional.

O objetivo geral é comparar os níveis de assimilação propiciados por dois diferentes procedimentos adotados, trabalhando-se conteúdos programáticos idênticos na disciplina de Administração de Materiais e Bens Patrimoniais nas IES UniFMU e Luzwell. Investigou-se se, para um dado conteúdo programático, o procedimento centrado no aluno $\left(\mathrm{P}_{2}\right)$ promoveu níveis de assimilação diferentes do procedimento centrado no professor $\left(\mathrm{P}_{1}\right)$.

Para avaliar os níveis de assimilação propiciados pelos dois procedimentos, recorreu-se a quatro modalidades de avaliação (Bloom, 1956; Cronbrach, 1963 como citado em Chaves, 2003; Tyler, 1989):

1) Diagnóstica - antecede o programa, diagnosticando o nível de conhecimento prévio.

2) Formativa - realizada no final de cada aula; mede a assimilação de conteúdos.

3) Somativa - imediatamente no término do programa avalia os resultados acumulados.

4) De retenção - posterior à somativa, mede resultados retidos ao longo do tempo.

Os dados gerados pelas quatro modalidades (avaliações objetivas) constituem a base de dados primários coletados no experimento. 
Foram definidas quatro hipóteses a serem testadas:

- $\mathrm{H}_{0-1}$ : O nível de acertos na avaliação somativa é igual ao nível de acertos na avaliação diagnóstica para os dois procedimentos.

- $\mathrm{H}_{0-2}$ : O nível de acertos na avaliação somativa é igual para os dois procedimentos.

- $\mathrm{H}_{0-3}$ : O nível de acertos nas avaliações formativas é igual para os dois procedimentos.

- $\mathrm{H}_{0-4}$ : O nível de acerto na avaliação de retenção é igual para os dois procedimentos.

\section{Método de Pesquisa}

O estudo foi realizado em duas instituições privadas de ensino superior em Administração, na cidade de São Paulo. Trata-se de uma pesquisa descritiva não probabilística em função da escolha intencional da população. Os dados foram produzidos por experimentação com quatro grupos de alunos, sendo dois os grupos de controle (procedimento $\mathrm{P}_{1}$ centrado no professor) e dois os grupos experimentais (procedimento $\mathrm{P}_{2}$ centrado nos alunos). $\mathrm{O}$ experimento foi conduzido durante parte do programa da disciplina, concentrando-se no tópico Planejamento de Recursos Materiais [MRP]. O tratamento dos dados fez uso da técnica estatística de emparelhamento e do teste de soma de classificações de Wilcoxon para exame das diferenças entre duas medianas.

\section{Descrição do ExPERIMENTO}

O estudo foi realizado no período de 6 de fevereiro a 4 de junho de 2004 . Trabalhou-se com 201 alunos regularmente matriculados na disciplina de Administração de Materiais e Bens Patrimoniais nas faculdades UniFMU e Luzwell conforme descrito na Tabela 1:

. 155 alunos de Administração de Empresas das Faculdades Luzwell, divididos alfabeticamente em duas turmas de 77 e 78 alunos.

. 46 alunos de Administração Hoteleira das Faculdades UniFMU, divididos alfabeticamente em duas turmas de 24 e 19 alunos. 
Tabela 1: Horários para os Grupos $P_{1}$ e $P_{2}$

\begin{tabular}{|c|c|c|c|c|c|c|}
\hline Faculdades & Horário & $2^{\mathrm{a}}$ & $3^{\mathrm{a}}$ & $4^{\mathrm{a}}$ & $5^{\mathrm{a}}$ & $6^{\mathrm{a}}$ \\
\hline \multirow{3}{*}{ Unifmu } & Manhã & & & $\mathrm{P} 1$ & & \\
\hline & Noite & & & & & $\mathrm{P} 2$ \\
\hline \multirow{3}{*}{ Luzwell } & Manhã & & & & & \\
\hline & Noite & P1 & & P2 & & \\
\hline
\end{tabular}

Destaca-se na Tabela 1 o cuidado no encadeamento dos grupos submetidos aos dois procedimentos. Nas duas primeiras aulas da semana ( $2^{\mathrm{a}}$ à noite e $4^{\mathrm{a}}$ pela manhã) foi adotado o procedimento $\mathrm{P}_{1}$. $\mathrm{O}$ procedimento $\mathrm{P}_{2}$ foi adotado a seguir (4a à noite $\mathrm{e}$ 6a. pela manhã). Procurou-se reduzir as eventuais interferências dos procedimentos. Nas duas instituições o estudo ocupou 14 horas do total de 40 horas previstas no programa, com bibliografia e conteúdos programáticos idênticos nas quatro turmas.

\section{Procedimentos Didáticos}

Para os dois procedimentos, os problemas de gestão foram desenvolvidos e estruturados para um restaurante de coletividades identificado como Restaurante Simulado. Segundo Fonseca (2002, pp. 17-28) restaurante de coletividades é orientado a atender a demanda alimentar dos funcionários da empresa que contratou este conceito, sendo seu cardápio menos variado e dirigido às necessidades nutricionais do quadro funcional. O conteúdo programático de cada aula foi contemplado com uma situação-problema apoiada no restaurante de coletividades.

O encadeamento das atividades e a formulação das situações-problema seguem o ciclo de aprendizagem vivencial proposto por Kolb (1984): experiência concreta, observação e reflexão sobre a experiência concreta, formação de conceitos abstratos e teste dos conceitos em situações novas. No restaurante simulado o aluno estará exposto a uma situação-problema que permite vivenciar uma experiência concreta, permitindo reflexão sobre ela. No momento das avaliações verifica-se a capacidade do participante em abstrair-se da situação vivenciada e aplicá-la a um novo problema em outro contexto.

A Tabela 2 descreve a sequiência de atividades de cada uma das sete aulas, o tempo designado para cada atividade nos dois procedimentos e o encadeamento lógico comparativo entre eles. No procedimento centrado no professor $\left(\mathrm{P}_{1}\right)$ destinaram-se dois terços do tempo total à exposição do conteúdo programático (80') e um terço para os alunos trabalharem individualmente na solução dos problemas propostos $\left(40^{\prime}\right)$. No procedimento centrado no aluno $\left(\mathrm{P}_{2}\right)$, metade do 
tempo foi dedicada a atividades em grupo (60') ou individual (prova) e o complemento à exposição de conteúdos (60').

\section{Tabela 2: Descrição da Sequiência de Atividades e Tempo Previstos para Cada Procedimento}

\begin{tabular}{|c|c|c|c|}
\hline \multicolumn{2}{|c|}{ Procedimento centrado no professor - $\mathrm{P}_{1}$} & \multicolumn{2}{|l|}{ Procedimento centrado no participante $-\mathrm{P}_{2}$} \\
\hline Tempo & Atividade & Atividade & Tempo \\
\hline \multirow[b]{2}{*}{$30^{\prime}$} & \multirow[b]{2}{*}{ Exposição da teoria pelo professor } & Exposição do problema pelo professor & $10^{\prime}$ \\
\hline & & $\begin{array}{l}\text { Cada grupo de alunos auto-organizados } \\
\text { estuda e propõe solução inicial para o } \\
\text { problema (solução 1) }\end{array}$ & 20 ' \\
\hline $10^{\prime}$ & Exposição do problema pelo professor & & \\
\hline 20 ' & $\begin{array}{l}\text { Alunos individualmente estudam o } \\
\text { problema e propõem solução }\end{array}$ & Exposição da teoria pelo professor & 30 ' \\
\hline \multirow[t]{2}{*}{$40^{\prime}$} & \multirow{2}{*}{$\begin{array}{l}\text { Professor apresenta a resolução } \\
\text { completa do problema e relaciona com } \\
\text { a teoria }\end{array}$} & $\begin{array}{l}\text { Cada grupo de alunos revisa o problema e } \\
\text { propõe a solução } 2\end{array}$ & $20^{\prime}$ \\
\hline & & $\begin{array}{l}\text { Professor apresenta a resolução completa do } \\
\text { problema e relaciona com a teoria }\end{array}$ & $20{ }^{\prime}$ \\
\hline \multirow[t]{2}{*}{20 ' } & $\begin{array}{l}\text { Aplicação de prova objetiva para } \\
\text { avaliação da assimilação de conteúdo }\end{array}$ & $\begin{array}{l}\text { Aplicação de prova objetiva para avaliação } \\
\text { da assimilação de conteúdo }\end{array}$ & 20 ' \\
\hline & Final da aula & Final da aula & \\
\hline
\end{tabular}

O procedimento $\mathrm{P}_{1}$ é iniciado com apresentação da teoria pelo professor (30'). Terminada esta etapa, apresenta-se a situação-problema (10') pertinente ao tópico programático abordado, dando-se tempo para que, individualmente, cada aluno elabore sua solução (20'). Em seguida o professor expõe a solução recomendada pela literatura para a situação-problema (40').

O procedimento $\mathrm{P}_{2}$ tem início com a exposição do problema (10') seguido da resolução da situação-problema pertinente ao tópico a ser desenvolvido. Os participantes, reunidos em grupos de quatro, propõem a solução inicial a partir de suas percepções e experiências prévias (20'). Esta solução 1 (provisória) é recolhida após 20 minutos. Na sequiência o professor expõe a teoria (30') O grupo de alunos revisa a solução 1 e propõe a solução 2, à luz da teoria apresentada $\left(20^{\prime}\right)$. O professor apresenta a resolução oficial (20').

Ao término de cada aula foi aplicada uma avaliação formativa idêntica em ambos os procedimentos, visando avaliar níveis de assimilação de conteúdos atingidos pelos diferentes métodos.

\section{Instrumentos de Avaliação}

Todos os participantes foram avaliados individualmente por meio de provas específicas, permitindo-se uso de calculadora, sem consulta a nenhum material 
de apoio. Na primeira aula de cada grupo destinou-se 60 minutos para a aplicação de uma prova denominada "Instrumento de Medição de Assimilação de Conteúdos [IMAC]", usada como avaliação diagnóstica dos participantes. Composta de dez questões de múltipla escolha adaptadas dos livros-texto, além das alternativas de (a) a (e), contava com as alternativas (f) e (g):

Alternativa (f): Não me recordo de ter estudado nada relacionado com o tópico em questão.

Alternativa (g): Recordo-me de ter estudado o assunto, mas não sei como resolver.

Qual disciplina:

Foram promovidas cinco avaliações formativas destinando-se 20 minutos para sua realização no final de cada encontro. Cada avaliação foi composta de cinco questões estruturadas em três níveis de dificuldade. As duas primeiras questões são resolvidas, aplicando-se regras e fórmulas. A terceira e quarta questões exigem interpretação e avaliação de dados para a elaboração da solução. A quinta questão envolve julgamento a partir da interpretação de resultados.

Uma semana após o encerramento do programa, elaborou-se a avaliação somativa, utilizando-se do IMAC. A sequiência das questões e os dados numéricos da primeira versão foram modificados e as alternativas (f) e (g) excluídas.

Após 6 semanas do término do programa, aplicou-se a prova de final de semestre, destinando-se 120 minutos para sua resolução. Composta de 20 questões, sendo dez do IMAC, com seqüência e dados modificados e outras dez questões dos demais itens desenvolvidos durante o semestre, permitiu medir a retenção dos conteúdos.

\section{Resultados das Avaliações}

A Tabela 3 representa o desempenho dos participantes na avaliação diagnóstica nas duas instituições. A coluna acertos possíveis representa quantidade de participantes multiplicada pelo número de questões. Respostas certas nesta avaliação não ultrapassaram duas tentativas com elevada incidência de respostas f. Os resultados indicam que os conteúdos contemplados no $\mathrm{IMAC}_{1}$ são desconhecidos para praticamente todos os participantes. Este fato é demonstrado pela coluna Respostas certas da Tabela 3 . 


\section{Tabela 3: Registro de Desempenho dos Alunos da Diferentes Instituições no IMAC}

\begin{tabular}{|c|c|c|c|c|c|c|}
\hline \multirow{2}{*}{ Faculdade } & Procedimento & $\begin{array}{c}\text { Total de } \\
\text { alunos }\end{array}$ & $\begin{array}{c}\text { Resposta } \\
\text { "f" }\end{array}$ & $\begin{array}{c}\text { Respostas } \\
\text { certas }\end{array}$ & $\begin{array}{c}\text { Respostas } \\
\text { erradas }\end{array}$ & $\begin{array}{c}\text { Acertos } \\
\text { possíveis }\end{array}$ \\
\hline \multirow{2}{*}{ UniFMU } & $\mathrm{P}_{1}$ & 24 & 201 & 1 & 38 & 240 \\
\cline { 2 - 7 } & $\mathrm{P}_{2}$ & 19 & 167 & 1 & 22 & 190 \\
\hline \multirow{2}{*}{ Luzwell } & $\mathrm{P}_{1}$ & 78 & 640 & 0 & 140 & 780 \\
\cline { 2 - 7 } & $\mathrm{P}_{2}$ & 77 & 658 & 2 & 110 & 770 \\
\hline
\end{tabular}

A Tabela 4 indica o índice de acerto percentual para cada questão nas duas instituições, para as cinco avaliações formativas. Cada avaliação pode totalizar no máximo 5 pontos. As avaliações formativas fornecem fotos de assimilação dos conteúdos programáticos a cada tópico desenvolvido. Os índices de acertos nas cinco avaliações formativas foram superiores para o procedimento centrado no participante $\left(\mathrm{P}_{2}\right)$.

Tabela 4: Percentual de Acertos nas Avaliações Formativas

\begin{tabular}{|c|c|c|c|c|c|c|c|c|c|c|c|}
\hline \multirow{3}{*}{$\begin{array}{l}\text { Avaliação } \\
\text { Formativa }\end{array}$} & \multirow{3}{*}{$\begin{array}{l}\text { Procedi- } \\
\text { mento }\end{array}$} & \multicolumn{10}{|c|}{ Percentual de acertos para cada questão da avaliação formativa } \\
\hline & & \multicolumn{5}{|c|}{ Luzwell } & \multicolumn{5}{|c|}{ UniFMU } \\
\hline & & $1^{\mathrm{a}}$ & $2^{\mathrm{a}}$ & $3^{\mathrm{a}}$ & $4^{\mathrm{a}}$ & $5^{\mathrm{a}}$ & $1^{\mathrm{a}}$ & $2^{\mathrm{a}}$ & $3^{\mathrm{a}}$ & $4^{a}$ & $5^{\text {a }}$ \\
\hline \multirow[t]{2}{*}{1} & $\mathrm{P}_{1}$ & 79,2 & 71,4 & 77,9 & 85,7 & 79,2 & 75,0 & 66,7 & 58,3 & 58,3 & 50,0 \\
\hline & $\mathrm{P}_{2}$ & 93,5 & 89,6 & 92,2 & 96,1 & 88,3 & 90,9 & 95,5 & 81,8 & 77,3 & 72,7 \\
\hline \multirow[t]{2}{*}{2} & $\mathrm{P}_{1}$ & 78,4 & 71,6 & 86,5 & 91,9 & 90,5 & 72,7 & 77,3 & 68,2 & 68,2 & 50,0 \\
\hline & $\mathrm{P}_{2}$ & 93,3 & 88,0 & 90,7 & 100 & 96,0 & 90,5 & 95,2 & 90,5 & 90,5 & 81,0 \\
\hline \multirow[t]{2}{*}{3} & $\mathrm{P}_{1}$ & 76,3 & 68,4 & 81,6 & 73,7 & 77,6 & 65,2 & 69,6 & 65,2 & 47,8 & 56,5 \\
\hline & $\mathrm{P}_{2}$ & 94,7 & 89,3 & 92,0 & 88,0 & 90,7 & 81,8 & 81,8 & 90,9 & 72,7 & 72,7 \\
\hline \multirow[t]{2}{*}{4} & $P_{1}$ & 74,3 & 68,9 & 82,4 & 91,9 & 83,8 & 68,2 & 72,7 & 72,7 & 63,6 & 63,6 \\
\hline & $\mathrm{P}_{2}$ & 96,0 & 86,7 & 93,3 & 94,7 & 93,3 & 85,7 & 81,0 & 90,5 & 90,5 & 95,2 \\
\hline \multirow[t]{2}{*}{5} & $\mathrm{P}_{1}$ & 68,4 & 65,8 & 73,7 & 84,2 & 64,5 & 58,3 & 62,5 & 70,8 & 50,0 & 62,5 \\
\hline & $\mathrm{P}_{2}$ & 88,3 & 83,1 & 92,2 & 93,5 & 83,1 & 72,7 & 90,9 & 90,9 & 72,7 & 86,4 \\
\hline
\end{tabular}

A Tabela 5 exibe os resultados obtidos na avaliação somativa, realizada no término do conjunto de 5 aulas sobre Planejamento de Recursos Materiais [MRP]. Observa-se a quantidade de acertos e o percentual que este valor representa na população estudada. Os resultados indicam que na Luzwell 5,3\% dos alunos submetidos ao procedimento $\mathrm{P}_{1}$ obtiveram nota igual ou superior a sete contra $48,1 \%$ para $\mathrm{P}_{2}$. Na UniFMU tem-se que $18,1 \%$ e $23,8 \%$ respectivamente para os métodos $\mathrm{P}_{1}$ e $\mathrm{P}_{2}$. 
Tabela 5: Total de Acertos na Avaliação Somativa

\begin{tabular}{|c|c|c|c|c|c|c|c|c|}
\hline $\begin{array}{c}\text { Quantidade de } \\
\text { acertos }\end{array}$ & \multicolumn{5}{|c|}{ Luzwell } & \multicolumn{5}{c|}{ UniFMU } \\
\hline & \multicolumn{2}{|c|}{$\mathrm{P}_{1}$} & \multicolumn{2}{c|}{$\mathrm{P}_{2}$} & \multicolumn{2}{c|}{$\mathrm{P}_{1}$} & \multicolumn{2}{c|}{$\mathrm{P}_{2}$} \\
\hline & quantidade & $\%$ & quantidade & $\%$ & quantidade & $\%$ & quantidade & $\%$ \\
\hline & 0 & 0 & 0 & 0 & 0 & 0 & 0 & 0 \\
\hline 1 & 0 & 0 & 0 & 0 & 1 & 4,5 & 0 & 0 \\
\hline 2 & 1 & 1,3 & 0 & 0 & 2 & 9,0 & 0 & 0 \\
\hline 3 & 1 & 1,3 & 1 & 1,3 & 1 & 4,5 & 1 & 4,8 \\
\hline 4 & 29 & 39,1 & 1 & 1,3 & 3 & 13,7 & 1 & 4,8 \\
\hline 5 & 22 & 30,0 & 19 & 25,3 & 8 & 36,5 & 5 & 23,7 \\
\hline 6 & 17 & 23,0 & 18 & 24,0 & 3 & 13,7 & 9 & 42,9 \\
\hline 7 & 3 & 4,0 & 22 & 29,4 & 4 & 18,1 & 2 & 9,5 \\
\hline 8 & 1 & 1,3 & 8 & 10,7 & 0 & 0 & 3 & 14,3 \\
\hline 9 & 0 & 0 & 6 & 8,0 & 0 & 0 & 0 & 0 \\
\hline 10 & 0 & 0 & 0 & 0 & 0 & 0 & 0 & 0 \\
\hline
\end{tabular}

Observando-se os resultados da Tabela 5 ilustrados nas Figuras 2 e 3, nota-se que em ambas as instituições o desempenho superior foi atingido pelos alunos submetidos ao procedimento $\mathrm{P}_{2}$.

Figura 2: Desempenho na Luzwell em Avaliação Somativa

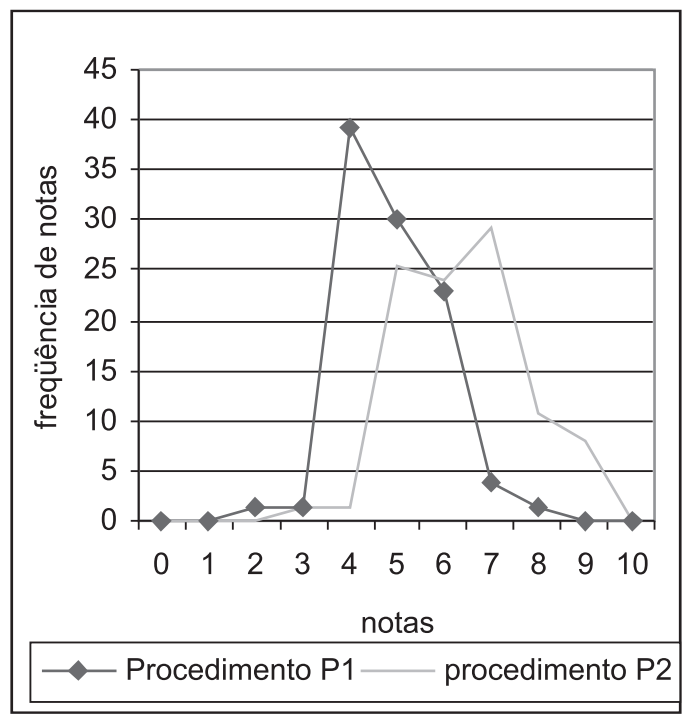




\section{Figura 3: Desempenho na UniFMu em Avaliação Somativa}

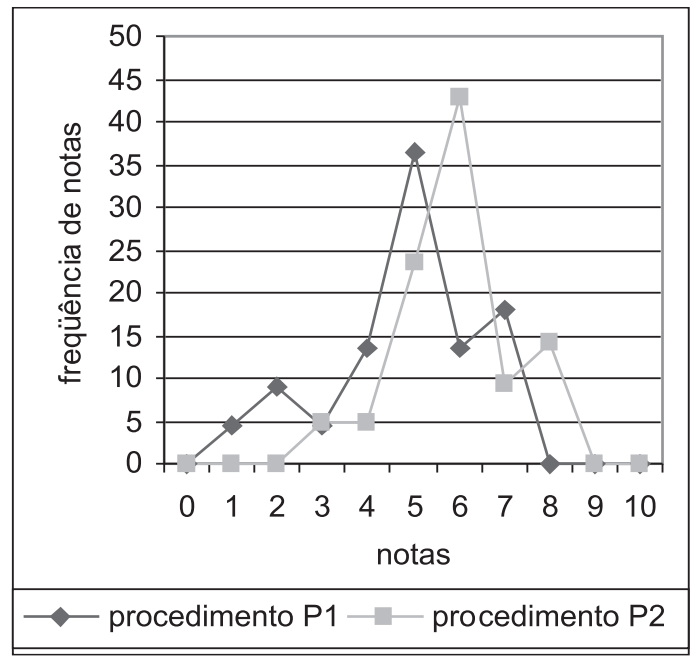

A Tabela 6 apresenta os resultados da avaliação de retenção realizada 6 semanas após a conclusão das aulas sobre MRP. Para alunos da Luzwell submetidos ao procedimento $\mathrm{P}_{1}$, apenas $1,4 \%$ obtiveram notas iguais ou superiores a sete, enquanto para o procedimento $\mathrm{P}_{2}$ este número atingiu 38,7\%. Na UniFMU têmse respectivamente $9,0 \%$ e $23,8 \%$.

Observa-se que, independentemente do procedimento ou instituição, houve queda no número de acertos da avaliação somativa para a de retenção; e que, embora se tenham índices elevados de reprovação nestas condições, os melhores resultados comparativamente foram conseguidos pelos alunos submetidos ao procedimento $\mathrm{P}_{2}$ nas duas instituições.

Tabela 6: Total de Acertos Obtidos pelos Participantes na Avaliação de Retenção

\begin{tabular}{|c|c|c|c|c|c|c|c|c|}
\hline No. de acertos & \multicolumn{4}{|c|}{ Luzwell } & \multicolumn{4}{c|}{ UniFMU } \\
\hline & \multicolumn{2}{|c|}{$\mathrm{P}_{1}$} & \multicolumn{2}{c|}{$\mathrm{P}_{2}$} & \multicolumn{2}{c|}{$\mathrm{P}_{1}$} & \multicolumn{2}{c|}{$\mathrm{P}_{2}$} \\
\hline & quantidade & $\%$ & quantidade & $\%$ & Quantidade & $\%$ & quantidade & $\%$ \\
\hline 0 & 0 & 0 & 0 & 0 & 1 & 4,5 & 0 & 0 \\
\hline 1 & 0 & 0 & 0 & 0 & 2 & 9,1 & 2 & 9,5 \\
\hline 2 & 3 & 4,1 & 2 & 2,7 & 4 & 18,2 & 2 & 9,5 \\
\hline 3 & 8 & 10,8 & 2 & 2,7 & 3 & 13,7 & 3 & 14,3 \\
\hline 4 & 31 & 41,8 & 3 & 4,0 & 3 & 13,7 & 2 & 9,5 \\
\hline 5 & 20 & 27,0 & 19 & 25,3 & 5 & 22,7 & 4 & 19,1 \\
\hline 6 & 11 & 14,9 & 20 & 26,6 & 2 & 9,1 & 3 & 14,3 \\
\hline 7 & 1 & 1,4 & 19 & 25,3 & 1 & 4,5 & 2 & 9,5 \\
\hline 8 & 0 & 0 & 8 & 10,7 & 1 & 4,5 & 3 & 14,3 \\
\hline 9 & 0 & 0 & 2 & 2,7 & 0 & 0 & 0 & 0 \\
\hline 10 & 0 & 0 & 0 & 0 & 0 & 0 & 0 & 0 \\
\hline
\end{tabular}


As Figuras 4 e 5 ilustram os resultados da avaliação de retenção. Comparandose tais resultados com as Figuras 2 e 3, nota-se que houve perda de desempenho. Apesar disso constata-se que o procedimento $\mathrm{P}_{2}$ manteve, relativamente, resultados superiores.

Figura 4: Desempenho na Luzwell em Avaliação de Retenção

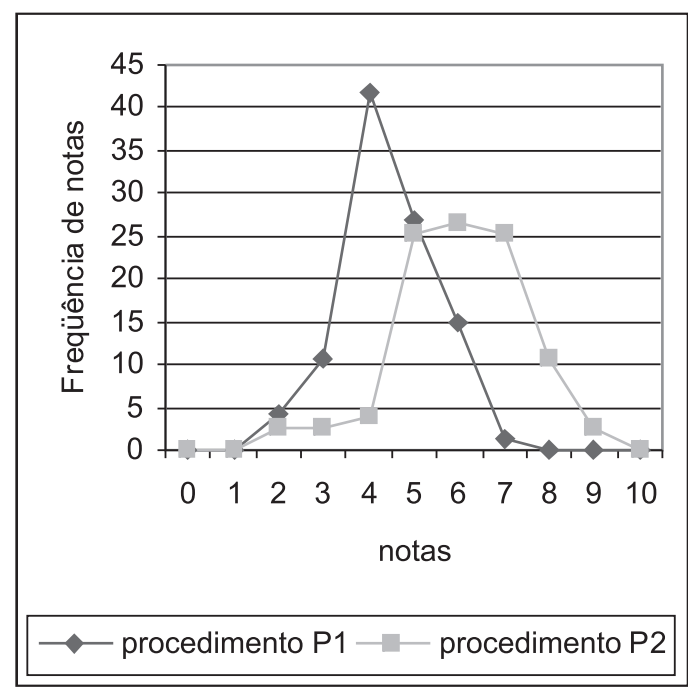

Figura 5: Desempenho na UNiFMU em Avaliação de Retenção

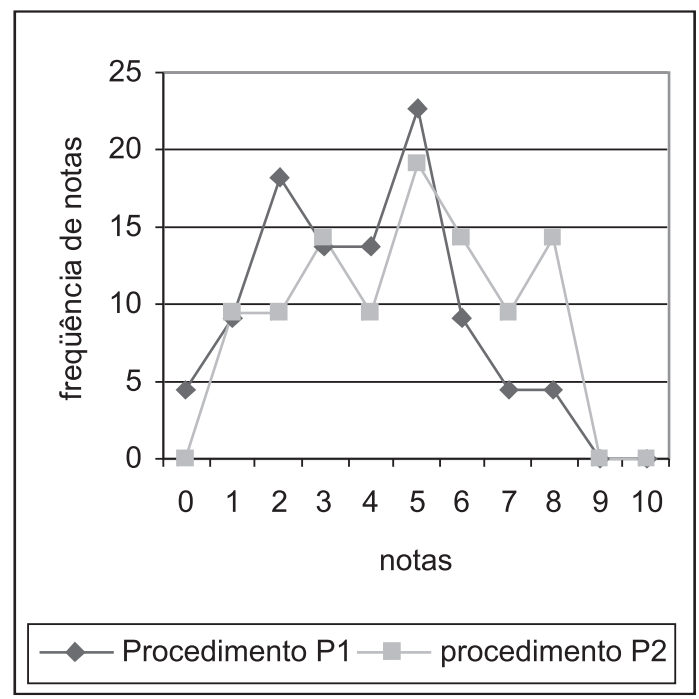




\section{Teste de Hipóteses}

Para as avaliações formativas, testou-se, em cada encontro, a igualdade do nível de acertos obtidos pelos diferentes procedimentos, nas duas instituições, conforme ilustra a Tabela 7.

Tabela 7: Teste de Hipótese Realizado para as Avaliações Formativas

\begin{tabular}{|c|}
\hline Teste 2 \\
\hline $\mathrm{H}_{0-2:} \mathrm{F}_{1 \mathrm{i}}=\mathrm{F}_{2 \mathrm{i}}$ \\
\hline
\end{tabular}

Legenda: F1i,2i - Resultados das avaliações formativas.

A Tabela 8 apresenta os valores limites da região de rejeição da hipótese nula para níveis de significância de $1 \%$ e os resultados obtidos no teste da soma de classificações de Wilcoxon para diferenças entre medianas, bicaudal para as duas instituições participantes do experimento. Os resultados registrados indicam que para o nível de significância testado (1\%) ocorreu rejeição da hipótese nula em todas as avaliações formativas. Este fato sugere que o nível de assimilação de conteúdos promovido pelo procedimento $\mathrm{P}_{2}$ foi, em todos os casos observados, superior ao $\mathrm{P}_{1}$ em ambas as instituições.

\section{Tabela 8: Resultados no Teste de Soma de Wilcoxon para Luzwell e UniFMU}

\begin{tabular}{|c|c|c|c|c|c|}
\hline $\begin{array}{c}\text { Provas } \\
\text { Luzwell }\end{array}$ & $\begin{array}{c}\text { Provas } \\
\text { UniFMU }\end{array}$ & $\begin{array}{c}\text { Limite inferior da região } \\
\text { de aceitação }(\alpha=1 \%)\end{array}$ & $\begin{array}{c}\text { Limite superior da região } \\
\text { de aceitação }(\alpha=1 \%)\end{array}$ & \\
\hline & & & $-2,575$ & $+2,575$ & \\
\hline & & Rejeita $\mathrm{H}_{0}$ & Aceita $\mathrm{H}_{0}$ & Aceita $\mathrm{H}_{0}$ & Rejeita $\mathrm{H}_{0}$ \\
\hline & 1 & & & & 2,67 \\
\hline & 2 & $-3,30$ & & & \\
\hline & 3 & & & & 3,61 \\
\hline & 4 & $-4,49$ & & & \\
\hline & 5 & $-3,89$ & & & \\
\hline 1 & & $-4,21$ & & & $+4,41$ \\
\hline 2 & & & & & $+2,90$ \\
\hline 3 & & & & & \\
\hline 4 & & & & & $+2,88$ \\
\hline 5 & & & & & $+2,88$ \\
\hline
\end{tabular}




\section{Discussão dos Resultados na Avaliação Somativa}

A Tabela 9 mostra os testes realizados na avaliação somativa. A eventual rejeição da hipótese nula permitiria afirmar que procedimentos diferentes propiciaram assimilação diferente de conteúdos programáticos.

Tabela 9: Teste de Hipótese Realizado para Avaliação Somativa

Teste 3

$$
\mathrm{H}_{0-3}: \mathrm{D}_{1}=\mathrm{D}_{2}
$$

Legenda: F1i,2i - Resultado das avaliações formativas.

A realização do teste de soma de classificações de Wilcoxon para diferenças de medianas bicaudal aplicado aos resultados obtidos nos procedimentos $\mathrm{P}_{1}$ e $\mathrm{P}_{2}$ em cada instituição, gerou como resultados $-6,65$ para a Luzwell e $+7,20$ para a UniFMU, rejeitando-se a hipótese nula de igualdade de médias nas duas instituições com $\alpha=1 \%$. Tais resultados sugerem maior nível de assimilação de conteúdos para o procedimento experimental centrado nos participantes $\left(\mathrm{P}_{2}\right)$.

\section{Discussão dos Resultados na Avaliação de Retenção}

Realizada seis semanas após o encerramento do programa, o teste bicaudal de soma de classificações de Wilcoxon para diferenças de medianas gerou como resultados $-4,85$ para a Luzwell e $+6,31$ para a UniFMU, permitindo a rejeição da hipótese nula para as duas instituições, para nível de significância de $1 \%$. Estes resultados indicam melhor desempenho para os participantes do procedimento centrado no aluno $\left(\mathrm{P}_{2}\right)$ nas duas instituições.

Na avaliação somativa na Luzwell, observou-se que 5,3\% dos alunos submetidos ao procedimento centrado no professor $\left(\mathrm{P}_{1}\right)$ alcançaram notas iguais ou superiores a sete. No grupo $\mathrm{P}_{2}$ este indicador atingiu 48,1\%. Na UniFMU, 18,1\% dos estudantes participantes do procedimento $\mathrm{P}_{1}$ alcançaram nota igual ou superior a sete, e $23,8 \%$ no procedimento $\mathrm{P}_{2}$.

$\mathrm{Na}$ avaliação de retenção dos alunos da Luzwell, submetidos ao procedimento $\mathrm{P}_{1}$, apenas $1,4 \%$ deles obtiveram notas iguais ou superiores a sete, enquanto para o procedimento $\mathrm{P}_{2}$ este número atingiu 38,7\%. Na UniFMU, 9,0\% dos estudantes 
participantes do procedimento $\mathrm{P}_{1}$ conseguiram nota igual ou superior a sete, e $23,8 \%$ para o procedimento $\mathrm{P}_{2}$.

\section{Conclusões}

Os conteúdos programáticos eram novos para a totalidade dos alunos, conforme indica a avaliação diagnóstica. A avaliação diagnóstica revelou desconforto de alguns participantes quanto ao uso de modelos matemáticos na resolução de problemas ligados ao planejamento de recursos materiais. Esta preocupação acaba interferindo no modo de como o aluno participa da aula. Observa-se que este fica muito mais preocupado com o como resolver determinado exercício do que por que resolver e o que representam os resultados obtidos. Apesar do aparente constrangimento gerado por este tipo de avaliação, sua contribuição foi clara para a identificação das dificuldades que seriam enfrentadas no desenvolvimento do programa.

Sem abrir mão dos conteúdos oficiais, observou-se que trabalhar com situaçõesproblema (exercícios, exemplos) de complexidade matemática variada pode auxiliar na orientação do foco do aluno. Os modelos matemáticos devem ser entendidos pelos futuros administradores como instrumentos que podem melhorar as práticas de gestão. Situações de menor complexidade tornam os cálculos mais intuitivos, demandam tempo menor e podem ser orientadas à interpretação dos resultados e seus efeitos no processo de tomada de decisão. Ambos os procedimentos demonstraram o avanço da assimilação de conteúdos, o que se pode constatar nos três tipos de avaliações adotados: formativa, somativa e de retenção.

Respeitados o conteúdo programático e o tempo total disponível em cada aula, observa-se que a única diferença entre os procedimentos está no encadeamento das atividades. $\mathrm{O}$ aluno colocado no centro do processo $\mathrm{P}_{2}$ tornou-se agente com o professor e ambos somaram esforços para construir conhecimentos.

Os procedimentos centrado no professor e centrado no participante contribuíram para a assimilação de conteúdos programáticos; o centrado no participante promoveu, de forma recorrente, desempenho superior nas avaliações formativas, somativa e de retenção.

A diferença mais acentuada de desempenho foi observada na avaliação de retenção, sugerindo que os alunos submetidos ao procedimento $\left(\mathrm{P}_{2}\right)$ retiveram por mais tempo os conceitos e modelos. Estudos complementares, replicando-se o experimento aqui descrito, modificando-se os exercícios sobre o tema, e aplicandoos após um longo espaço de tempo da conclusão da disciplina, poderão contribuir 
para revelar quais os níveis de assimilação e retenção que os diferentes procedimentos podem promover no longo prazo.

\section{CONTRIBUiçÕES}

$\mathrm{O}$ estudo buscou destacar o papel do aluno na aprendizagem e mostrar a utilidade de se incluir avaliações durante o processo educacional, mensurando em bases comparativas a evolução do processo e o aproveitamento dos alunos. Em lugar de avaliar unicamente o resultado final (avaliação somativa), levantaram-se dados diagnósticos de acompanhamento do processo em todo seu decurso (avaliações diagnóstica e formativas). Nesta abordagem a tradicional avaliação de encerramento foi substituída por criterioso acompanhamento do processo em todo seu desenvolvimento. A avaliação final de resultados passou a ser a de retenção, teste aplicado seis semanas após o término do programa objeto deste estudo.

\section{Limitações e Proposição para Novos Estudos}

Os resultados aqui reportados devem ser replicados por diferentes professores em diferentes disciplinas, com número maior de turmas em diferentes instituições para aprofundamento da pesquisa, validação dos instrumentos e disseminação das idéias ainda restritas a um grupo reduzido de pesquisadores. Além disso, para avançar os estudos, parece adequado ampliar a quantidade de questões do IMAC, trabalhando-se com diferentes níveis de complexidade para um diagnóstico mais amplo do perfil de conhecimento dos participantes. Este expediente poderá orientar a formação de grupos de trabalho e promover um refinamento na adaptação do procedimento a diferentes públicos. Estudos sobre formas alternativas de composição de grupos de trabalho poderiam contribuir significativamente para a prática educacional sempre que as atividades em grupo pudessem ser adotadas para promover a aprendizagem.

Cooper e Schindler (2003, pp. 322-337) afirmaram que experimentos verdadeiros devem trabalhar com grupos de controle e experimental gerados aleatoriamente, permitindo comparação por meio de testes estatísticos. Grupos formados por ordem alfabética são considerados por Wonnacott e Wonnacott (1985, pp. 3-14) aleatórios. $\mathrm{Na}$ Luzwell os grupos foram formados segundo este critério. Nas UniFMU as classes foram formadas em decorrência da escolha dos próprios participantes, muito 
antes de ser declarada a realização da pesquisa. Eventuais variações nesta instituição devem ser pesquisados para futura validação do experimento.

Para pequenas amostras ou populações sem distribuição normal, Levine, Berenson e Sthepan (2000, pp. 382-383) recomendam o uso do teste de soma de classificações de Wilcoxon para diferenças entre duas medianas. Segundo os autores o método não-paramétrico adotado neste estudo é quase tão eficaz quanto sua contrapartida paramétrica, o teste $t$, recomendado para populações com distribuição normal.

\section{Artigo recebido em 26.05.2006. Aprovado em 21.12.2006.}

\section{ReferênCIAS Bibliográficas}

Anastasiou, L.G. C. (1998).

Metodologia do ensino superior. Curitiba: IBPEX.

Becker, F. (1993).

Epistemologia do professor. Petrópolis: Vozes.

Bedworth, D. D., \&

Bailey, J. E. (1982).

Integrated production control systems. New York: Wiley \& Sons.

Bertrand, W. M.,

Wortmann, J. C., \&

Wijngaard, J. (1990).

Production control: a structural and design oriented approach. New York: Elsevier.

Bloom, B. S. (1956).

Taxonomy of educational objectives: Book one. Cognitive domain. New York: Longman.

Brown, G., \&

Atkins, M. (1997).

Effective teaching in higher education. Londres: Routledge.
Bujold, N., \&

Saint-Pierre, H. (1996).

Style d'intervention pédagogique, relations affectives enseignantesétudiants et engagement par raport a la matière. Canadian Journal of Higher Education, 26(1), 75-107.

Burbidge, J. L. (1981).

Planejamento e controle da produção. São Paulo: Atlas.

Cardoso, S. H. (1998).

Educação médica à distância pela Internet. Informática médica, 1(5), 4555 .

Carneiro, A. L. M. (2002).

O olhar dos egressos do curso superior de tecnologia em hotelaria sobre sua formação. Dissertação de mestrado não-publicada. Pontifícia Universidade Católica de São Paulo, São Paulo, SP, Brasil.

Cavalcanti, R. A. de (1999, julho). Andragogia: aprendizagem nos adultos. Revista de Clínica Cirúrgica da Paraíba, (6), Ano 4, 36-41. 
Chagas, A. T. R. (2002).

Dimensões do ensino em sala de aula de administração: um estudo sobre as preferências dos alunos. Tese de doutorado não-publicada. Universidade de São Paulo, São Paulo, SP, Brasil.

Chaves, S. M. (2003).

A avaliação da aprendizagem no ensino superior: realidade, complexidade e possibilidades. Tese de doutorado não-publicada. Universidade de São Paulo, São Paulo, SP, Brasil.

Cooper, D. R., \&

Schindler, P. S. (2003).

Métodos de pesquisa em administração. Porto Alegre: Bookman.

Correa, H. L.,

Gianesi, I. G. N., \&

Caon, M. (1999).

Planejamento, programação e controle da produção-MRP II / ERP conceitos, usos e implantação. São Paulo: Atlas.

Dias, M. A. P. (1997).

Administração de materiais: resumo da teoria, questões de revisão, exercícios e estudo de casos. São Paulo: Atlas.

Driscoll, M. P. (1994).

Psychology of learning for instruction. Boston: Allyn \& Bacon.

Eckstein, B. (1982).

Ensino superior: uma introdução prática. São Paulo: Pedagógica e Universitária.
Eilon, S. (1962).

Functions of production planning and control. New Jersey: Macmillan Company.

Feinstein, A. H. (2001).

The development and assessment of a foodservice instructional simulation technique (FIST). Journal of Hospitality \& Tourism Research, 25(4), 421-443.

Fonseca, M. T. (2002).

Tecnologias gerenciais de restaurantes. São Paulo: Senac.

Fouts, J. F., \&

Myers, R. E. (1992).

Classroom environments and middle school students views of science. Journal of Educational Research, 85(6), 356-361.

Gaither, N., \&

Frazier, G. (2002).

Administração da produção $e$ operações. São Paulo: Pioneira.

Gibbs, G. (1995).

Teaching students to learn. Buckingham: Open University.

Higgins, P.,

Roy, P., \&

Tierney, L. (1996).

Manufacturing planning and control systems. New York: Chapman \& HallUSA.

Keller, B. A., \&

Hirsch, C. R. (1998).

Students preferences for representations of functions. International Journal of Mathematical Education in Science and Tecnology, 29(1), 1-17. 
Kolb, D. A. (1984).

Experiential Learning: experience as the source of learning and development. Englewood Cliffs: Pretince-Hall.

Kuri, N.P. (1993).

As abordagens do processo ensinoaprendizagem: características gerais e metodológicas correspondentes. Tese de doutorado nãopublicada. Universidade de São Paulo, São Carlos, SP, Brasil.

Levine, D. M.,

Berenson, M. L., \&

Sthepan, D. (2000).

Estatística: teoria e aplicações. Rio de Janeiro: LTC.

Libâneo, J. C. (2004).

A didática e a aprendizagem do pensar e do aprender: a teoria históricocultural da atividade e a contribuição de Vasili Davydov. Revista Brasileira de Educação, (27), 5-24.

Luckesi, C. (1990).

Planejamento e avaliação na escola: articulação e necessária determinação ideológica $\left(\mathrm{N}^{\circ} 15\right.$, pp. 115-125). São Paulo: FDE.

Magee, J. F. (1958).

Production planning and inventory control. New York: McGrawHill.

Martins, G. A., \&

Passos, I. C. (2003).

Métodos de sucesso no ensino da Contabilidade. Anais do Congresso USP de Controladoria e Contabilidade, São Paulo, SP, Brasil, 3.
Martins, P. G., \&

Campos, P. R. (2000).

Administração de materiais e recursos patrimoniais. São Paulo: Saraiva.

Méndez, J. M. A. (2002).

Avaliar para conhecer, examinar para excluir. Porto Alegre: Artmed.

Moran, J. M., \&

Behrens, M. (2000).

Novas tecnologias e mediação pedagógica. Campinas: Papirus.

Moreira, D. (1996).

Administração da produção $e$ operações. São Paulo: Pioneira.

Moreira, D. (Org.). (1997).

Didática do ensino superior. São Paulo: Pioneira.

Morilha, P. O.,

Muritiba, S. N., \&

Casado, T. (2005, setembro)

Diferenças individuais dos estudantes e preferência por métodos de ensino em administração: uma aplicação dos tipos psicológicos de Jung. Anais do Encontro Nacional da Associação Nacional de Pós-Graduação e Pesquisa em Administração, Brasília, DF, Brasil, 29.

Morton, T. E., \&

Pentico, D. W. (1993).

Heuristic scheduling systems: with applications to production systems and project management. New York: Wiley \& Sons.

Pfeiffer, J. W., \&

Jones, E. J. (1980).

Coleção de exercícios estruturados para treinamento e educação. São Paulo: Intercultural. 
Plossl, G. W. (1985).

Production and inventory control. New Jersey: Prentice-Hall.

Pretto, F. N. (2006).

Pedagogia participativa na formação de administradores. Tese de doutorado não-publicada. Universidade de São Paulo, São Paulo, SP, Brasil.

Ramlow, D.E., \&

Wall, E. H. (1967).

Production planning and control. New Jersey: Prentice-Hall.

Rosenshine, B. (1995).

Advances in research on instruction. The Journal of Educational Research, 88(5), 262-268.

Saroyan, A., \&

Snell, L. S. (1997).

Variations in lecturing styles. Higher Education, 33(1), 85-104.

Sauaia, A. C. A. (1995).

Satisfação e aprendizagem em jogos de empresas. Tese de doutorado nãopublicada. Universidade de São Paulo, São Paulo, SP, Brasil.

Saul, A. M. (1988).

Avaliação emancipatória: desafio à teoria e a prática de avaliação e reformulação do currículo. São Paulo: Cortez.

Scheele, E. D.,

Westerman, W. L., \&

Wimmert, R. J. (1964).

Principles and design of production control Systems. New Jersey: Prentice-Hall.
Slack, N.,

Chambers, S.,

Harland, C.,

Harrison, A., \&

Johnston, R. (1999).

Administração da produção. São Paulo: Atlas.

Steffen, V., Jr. (1997).

A atual situação do ensino de engenharia no Brasil e perspectivas para o futuro. Revista de Ensino de Engenharia, 18, 36-39.

Teixeira, G. (1995).

Estado da arte da aprendizagem centrada no aluno. Recuperado em 19 fevereiro, 2007, de http:// www.serprofessoruniversitario.pro.br/ ler.php?modulo $=13 \&$ texto $=821$

Towne, D. M.,

Jong, T., \&

Spada, H. (Eds.). (1993).

Simulation-based experiential learning. Berlin: Springer-Verlag.

Tyler, R. (1989).

Educational evaluation: classic works. Boston: Klauwer Academic.

Vollmann, T. E.,

Berry, W. L., \&

Whybark, D. C. (1984).

Manufacturing planning and control systems. New York: Irwin/McGrawHill.

Vollmann, T. E.,

Berry, W. L., \&

Whybark, D. C. (1997).

Manufacturing planning and control systems. New York: Irwin/McGrawHill. 
Wang, M. C.,

Haertel, G. D., \&

Walberg, H. J. (1990).

What influences learning? A content analysis of review literature. Journal of Educational Research, 84, 30-43.
Wohl, R. (1995).

Manufacturing and services strategies. New Jersey: Prentice-Hall.

Wonnacott, R. J., \&

Wonnacott, T. H. (1985).

Fundamentos de estatística. Rio de Janeiro: LTC. 